\title{
Characteristic of Acoustic Emission Signal from Stress Corrosion Cracking in Low-Carbon Nitrogen-Enhanced Stainless Steel
}

\author{
Xuefeng Li, Yujiao Shao, Yuan Yu, Yin Zhang, and Runjie Shen* \\ College of Electronic and Information Engineering, Tongji University, \\ 4800 Cao'an Highway, Jiading Distrito, Shanghai 201804, P. R. China
}

(Received March 16, 2017; accepted June 8, 2017)

Keywords: nondestructive testing, acoustic emission, stress corrosion cracking, moment tensor solution

The problem of stress corrosion cracking (SCC), which causes sudden failure of metals subjected to stress in a high-temperature, high-pressure water environment, is studied. Acoustic emission (AE) monitoring is a promising method for detecting the initiation and propagation of SCC. In this study, pencil-lead breaks are used as AE signal sources to first validate the parameters for the verification of the finite element modeling of microcracking. Then, a simplified fracture propagation model of low-carbon nitrogen-enhanced (316LN) stainless steel is established on the basis of moment tensor theory. Finally, the inner connection between the energy release rate of the $\mathrm{AE}$ source and the morphological aspect of crack formation is analyzed. The results of modal analysis show that the amount of energy released by the growing crack is linearly proportional to crack depth. Moreover, their frequency characteristics are almost unchanged from the results of analysis by fast Fourier transform. Therefore, SCC initiation and propagation in 316LN stainless steel can be evaluated by this detection method. Moreover, this analysis method based on the energy release rate of the AE source can also be extended to almost all solid materials and structural crack detection.

\section{Introduction}

In the industrial production field, such as the power generation, petrochemical, and steel industries, a large number of pipelines and pressure vessels are constructed using austenitic stainless steel. In particular, low-carbon nitrogen-enhanced (316LN) stainless steel has been widely used because of its excellent corrosion resistance and good mechanical processing performance. ${ }^{(1-3)}$ However, stress corrosion cracking (SCC), pitting corrosion, corrosion fatigue, intergranular corrosion, and crevice corrosion, ${ }^{(4)}$ and all such phenomena are serious threats to production. ${ }^{(5-9)}$ Numerous investigations show that austenitic stainless steel is more sensitive to SCC in a high-temperature, high-pressure water environment. ${ }^{(10)}$ SCC is a cracking phenomenon of metal or alloy under the integrated effect of the media environment and tensile stress. For the past few years, many corrosion detection techniques based on the physical mechanical principle and electrochemical principle have been applied to corrosion protection in industry. Acoustic emission (AE) technologies, electrochemical noise (EN) technologies, guided wave ultrasonic

*Corresponding author: e-mail: shenrunjie@tongji.edu.cn

http://dx.doi.org/10.18494/SAM.2017.1604 
testing (GUT) technologies, and their combinations are the areas of greatest concern in SCC testing in the latest research. ${ }^{(11-15)}$ EN technologies have rapidly developed since they can enable the detection of various forms of corrosion, do not cause state changes in metallic materials during the corrosion process, and are amenable to continuous on-line monitoring. ${ }^{(16-18)}$ However, the lack of electrochemical kinetic information and unified evaluation criteria, and the inconsistency of results obtained by different signal processing methods make them unreliable. GUT technologies also are susceptible to high-temperature, high-pressure, and strong-radiation environments. AE technologies are extensively applied in health monitoring as a kind of dynamic nondestructive testing. ${ }^{(16,19-21)}$ Many research results show that there is a very close relationship between AE phenomena and material fracture. ${ }^{(22-27)}$ Therefore, AE monitoring has the potential to achieve remote real-time monitoring of the initiation and propagation of SCC.

AE phenomena refers to elastic wave phenomena in materials and structures produced by stress or fracture due to the rapid release of energy. The causes of AE sources are varied, such as dislocation movement, plastic deformation, and crack initiation and propagation. ${ }^{(28)}$ The ratio of $\mathrm{AE}$ signals generated by crack initiation to $\mathrm{AE}$ signals generated by single dislocation slip is at least 100. Theoretical calculations show that energy of crack propagation is 100 to 1000 times greater than that of crack initiation. Thus, the strength of AE signals generated by crack propagation is greater than crack initiation. ${ }^{(29)}$ Because the strength of AE signals generated by crack propagation is greater than that of signals generated by dislocation movement, plastic deformation, and crack initiation, the AE signal of crack propagation is most easily detected. With advantages such as sensitivity to geometry, integrity, and sensitivity to dynamic defects, AE monitoring techniques have been applied to monitor different types of corrosion. ${ }^{(30-32)}$ However, most of the research is focused on the characteristics of AE sources. To the best of our knowledge, no papers or reports on the specific relationship between crack propagation and $\mathrm{AE}$ signals have been published. In this study, cracks of various depths produced by stress corrosion cracking of $316 \mathrm{LN}$ stainless steel are identified by the energy release rates of AE sources. On the basis of the moment tensor theory in quantitative seismology, a mechanical model of AE source with extending crack depth has been formulated. The nonlinear finite element method (FEM) is used to obtain the AE signal data of the formation of cracks of various depths. In the process, the selection of the calculation parameters is fully discussed in order to ensure the accuracy of simulated results. Furthermore, pencillead breaks (PLBs) are used as AE signal sources to validate the parameters for the verification of the FEM modeling of microcracking. The PLB is a reproducible artificial AE source that is usually used to observe a microscopic displacement of the surface and causes an acoustic wave that propagates into the structure at the moment of material breakage. Here, the accuracy of a mechanical model of the AE source is verified by comparison between simulated and experimental results. Then, the FEM model of the crack produced in 316LN stainless steel is established, and the energy release rates of AE sources formed by SCC are analyzed. The energy release rates of AE sources are obtained under different crack depths. The results of modal analysis show that energy released by the growing crack is linearly proportional to crack depth. Therefore, the morphological aspects of crack formation can be determined from the amount of released energy. Moreover, their frequency characteristics are almost unchanged from the results of fast Fourier transform (FFT). This finding is very important in bandwidth selection and sensor selection. Therefore, this type of AE monitoring technique has the potential to achieve remote real-time monitoring of the initiation and propagation of SCC. 


\section{Model of AE Source}

$\mathrm{AE}$ generating mechanisms are associated with crack motions of kinetics and kinematics. The common characteristic of AE sources of crack initiation and propagation, crystalline phase dislocation, and fracture is the emergence of discontinuous displacement in the material. $\mathrm{AE}$ elastic waves produced by discontinuous displacement have a certain similarity to seismic waves produced by fault movements in an earthquake. Therefore, some mature theories in quantitative seismology can be adapted to the research of AE. Moment tensor theory provides a general mathematical representation of a point source that can be used to express fault motion in a microseismic source. ${ }^{(15)}$ Therefore, moment tensor theory can be cited to construct a mechanical model of a crack propagation AE source.

Generally, displacement $u_{n}(x, t)$ of a point in a continuous elastomer whose surface is defined as $\Sigma$ and volume is defined as $v$ is caused by the integrated action of body force $f$, traction force $T$, and discontinuous displacement $u$. The function $u_{n}(x, t)$ is the displacement at time $t$ of a point localized at $x$ in a continuous elastomer, is described by

$$
u_{n}(x, t)=\int_{-\infty}^{\infty} d \tau \iint_{\Sigma} m_{p q}(\xi, \tau) \frac{\partial G_{n p}(x, t-\tau ; \xi, 0)}{\partial \xi_{q}} d \Sigma(\xi)
$$

where $m_{p q}$ is the moment density tensor expressed as

$$
m_{p q}(\xi, \tau)=\left[u_{i}(\xi, \tau)\right] c_{i j p q}(\xi) v_{j}(\xi)
$$

In the above formulas, $\partial G_{n p}(x, t-\tau ; \xi, 0) / \partial \xi_{q}$ can be considered to be an equivalent couple acting on the surface $d \Sigma(\xi)$. Its strength is $m_{p q}(\xi, \tau), u_{i}(\xi, \tau)$ is the discontinuous displacement in the $i$ direction of surface $\xi$ at time $\tau, c_{i j p q}$ is the elastic constant of surface $\xi$, and $v_{j}(\xi)$ is the $j$ direction component of the surface normal unit vector.

However, the difficulty of solving $\partial G_{n p}(x, t-\tau ; \xi, 0) / \partial \xi_{q}$ by the theoretical method makes the calculation of the displacement time history $u_{n}(x, t)$ directly using Eqs. (1) and (2) impossible. If we construct a FEM model of a discontinuous displacement AE source, the displacement time history $u_{n}(x, t)$ can be obtained, avoiding the calculation of $\partial G_{n p}(x, t-\tau ; \xi, 0) / \partial \xi_{q}$. From Eq. (1), the transient displacement produced by discontinuous displacement $d_{x}$ of the microsurface is equivalent to the transient displacement produced by three pairs of couples $m_{x x} d \Sigma, m_{y y} d \Sigma$, and $m_{z z} d \Sigma$ loaded on the microsurface $d \Sigma 0$. Uniformly distribute three pairs of couples on the six microsurfaces of the microcrack unit $d x d y d z$.

$$
\begin{aligned}
& F_{y z}=\frac{m_{x x} d \Sigma}{d y d z d x}=\lambda+2 \mu \\
& F_{x z}=\frac{m_{y y} d \Sigma}{d x d z d y}=\mu \\
& F_{x y}=\frac{m_{z z} d \Sigma}{d x d y d z}=\mu
\end{aligned}
$$

Here, $\lambda$ and $\mu$ are Lamé constants. Thus, a mechanical model of a discontinuous displacement $\mathrm{AE}$ source is constructed to help solve the transient displacement produced by discontinuous displacement $d x$ in an object by FEM. 


\section{Experiment and Simulation of PLB}

In this section, the mechanical model of the AE source is validated by using the PLB as a reproducible artificial AE source. The experiment setup and FEM model are shown in Fig. 1. According to the velocities of the longitudinal and shear waves, to avoid reflection, a large plate of $316 \mathrm{LN}$ stainless steel of $50 \times 50 \times 0.8 \mathrm{~cm}^{3}$ is used in the experiment, and a two-dimensional FEM model of $50 \times 0.8 \mathrm{~cm}^{2}$ is used in the simulation. The material properties of 316LN stainless steel are shown in Table 1. An amplitude of $2.25 \mathrm{~N}$, which is in good agreement with that produced by the fracture of a $0.5-\mathrm{mm}$-diameter $2 \mathrm{H}$ pencil lead, is used. The piezoelectric sensor is manufactured by PengXiang Technology (Changsha, China) which has a resonance frequency of $300 \mathrm{kHz}$. It has a very high sensitivity and frequency response over the range of $80-400 \mathrm{kHz}$.

As shown in Fig. 2, FEM simulation and the experimental method are both used to analyze the AE signals of PLBs on a 316LN stainless-steel plate. The time-frequency analysis of AE signals is carried out by wavelet transform (WT) and its result is compared with the theoretical time-frequency distribution. The time-frequency distribution of PLB signals simulated by FEM is highly consistent with the theoretical one, which shows that the WT analysis of AE signals is effective and accurate and verifies the feasibility of FEM simulation on the propagation of PLB signals in the plate.

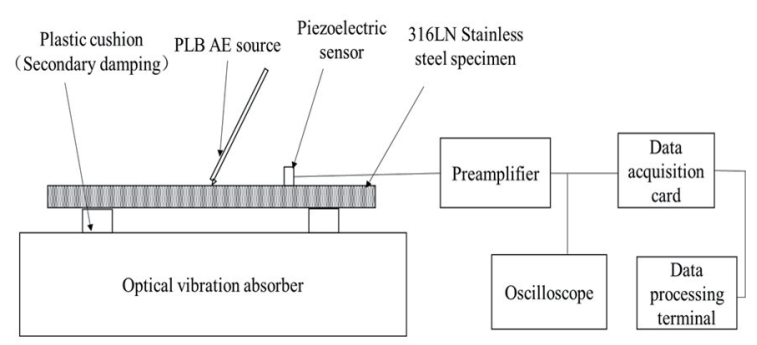

(a)

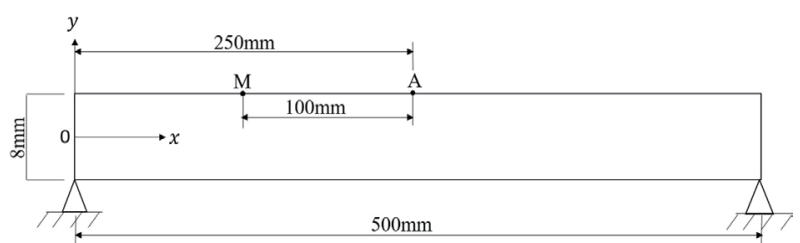

(c)

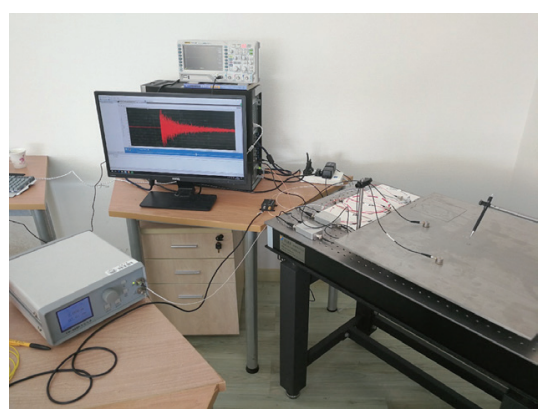

(b)

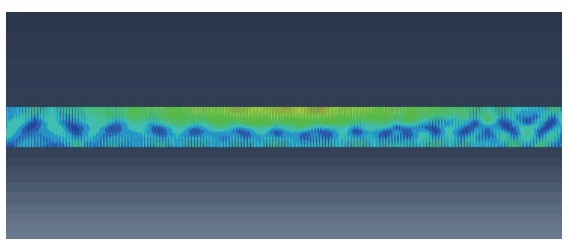

(d)

Fig. 1. (Color online) Experiment and simulation. (a) Test system, (b) photograph of experiment setup, (c) FEM model, and (d) example of simulation result.

Table 1

Material properties of $316 \mathrm{LN}$ austenitic stainless-steel plate.

\begin{tabular}{lclr}
\hline Young's modulus & $2.0 \times 10^{5}$ & Longitudinal waves $(\mathrm{mm} / \mu \mathrm{s})$ & 5.801 \\
Density $\left(\mathrm{kg} / \mathrm{m}^{3}\right)$ & $8.0 \times 10^{3}$ & Shear wave $(\mathrm{mm} / \mu \mathrm{s})$ & 3.100 \\
Poisson's ratio & 0.3 & Yield stress $(\mathrm{MPa})$ & 205 \\
\hline
\end{tabular}




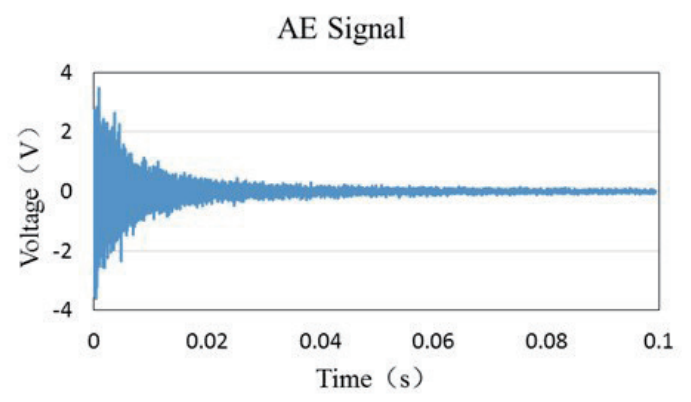

(a)

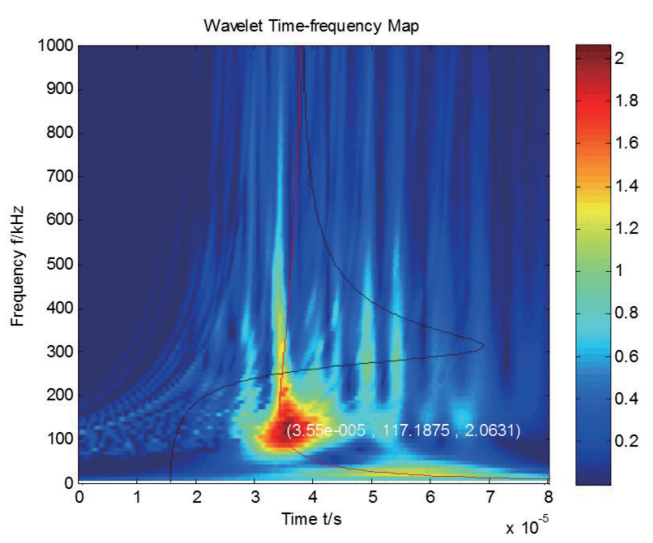

(c)

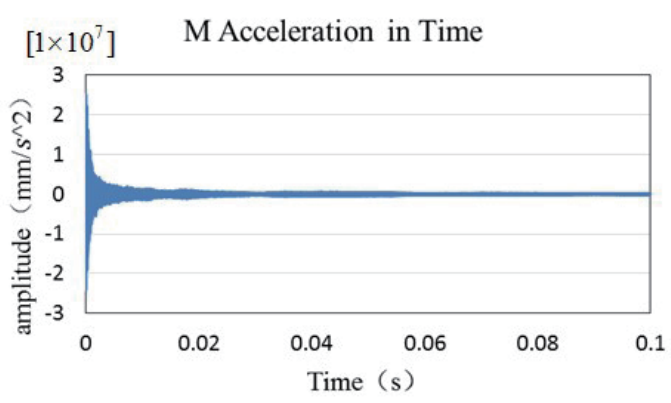

(b)

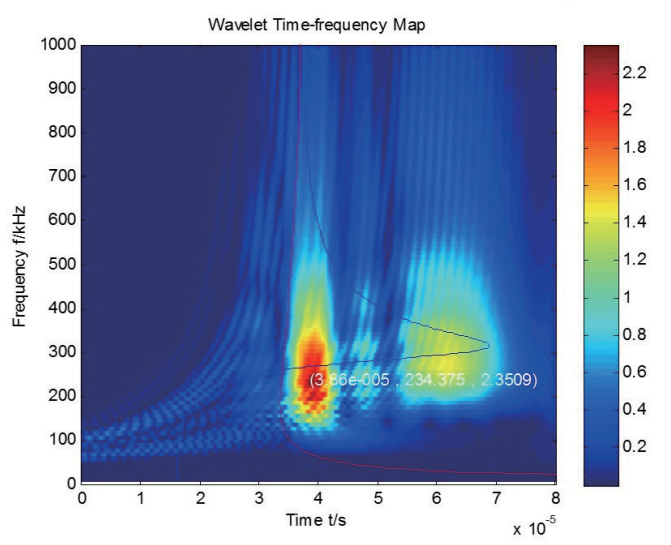

(d)

Fig. 2. (Color online) Results from experiments and simulations. (a) AE signal from sensor, (b) AE signal by FEM simulation, (c) time-frequency distribution of AE experimental signal, and (d) time-frequency distribution of AE simulation signal.

\section{Simulation of SCC}

In this section, AE signals generated by crack propagation in 316LN stainless steel at different crack depths have been studied. The FEM model of the AE signal in a 316LN stainless-steel plate is shown in Fig. 3(a). If we assume that the crack propagation in a unit plate width is uniform, the three-dimensional problem can be simplified to a two-dimensional plane strain problem. A square plate geometry was modeled with a length $10 \mathrm{~mm}$ along the $x$ direction and a thickness of $10 \mathrm{~mm}$ along the $y$ direction. The source (A) was positioned at $x=5 \mathrm{~mm}, y=10 \mathrm{~mm}$ on the surface of the plate. A receiver (M) was positioned at $x=5 \mathrm{~mm}, y=5 \mathrm{~mm}$ in the $1 / 2$ plate thickness. Three edges, excepting the bottom, are infinite elements to eliminate wave reflection. Figures 3(b)-3(d) show the AE wave-spreading process on the surface of the 316LN stainless-steel plate. The propagation time is consistent with the theoretical value of $0.69 \mu \mathrm{s}$, and the reflective energy is relatively weak.

Next, the energy release rates and the patterns of AE source are verified by comparison. When the crack depth of the plate is extended from 0.2 to $0.8 \mathrm{~mm}$ in $0.2 \mathrm{~mm}$ intervals, AE signals are 


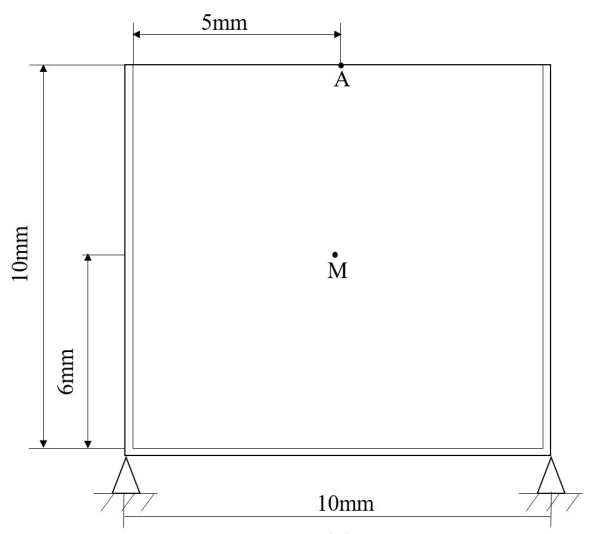

(a)

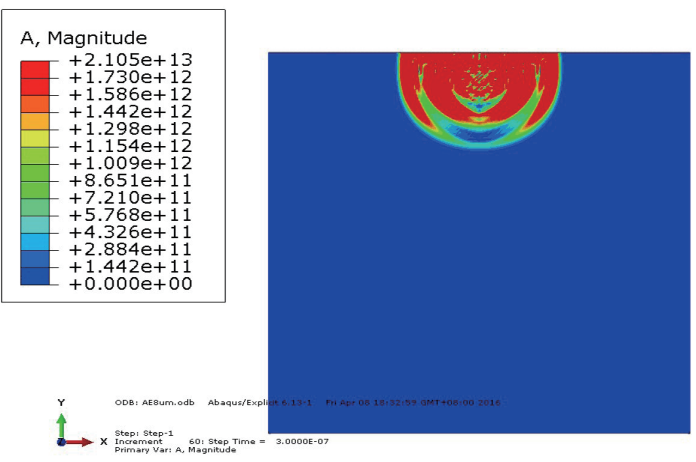

(c)

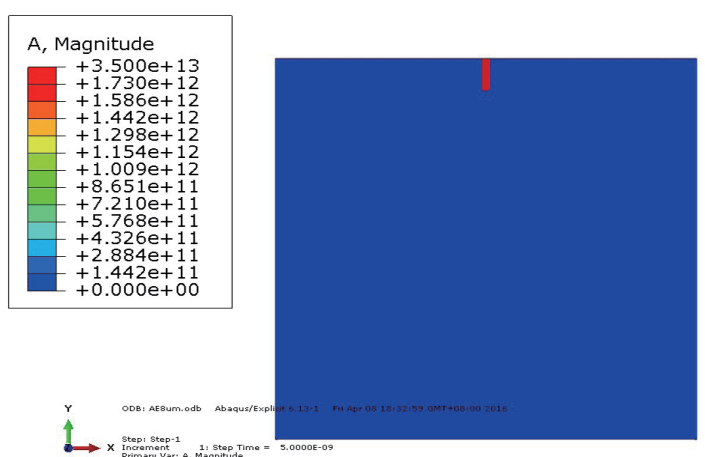

(b)

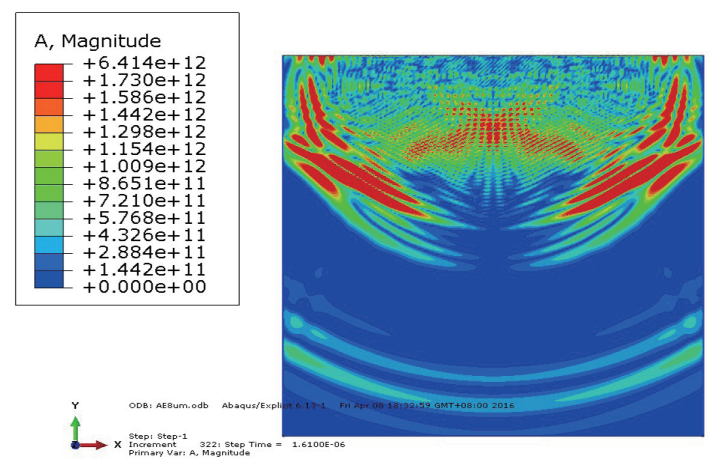

(d)

Fig. 3. (Color online) FEM simulation of SCC in 316LN stainless-steel plate. (a) FEM model, (b) AE wave transmission at $t=0.5 \mathrm{~ns}$, (c) AE wave transmission at $t=300 \mathrm{~ns}$, and (d) elastic wave transmission at $t=1610 \mathrm{~ns}$.

detected by the receiver (M), as shown in Fig. 4(a), and processed with a $20 \mathrm{kHz}$ high-pass filter, as shown in Fig. 4(b). Because of the increasing amplitude of the low-order mode at longer times, which corresponds to lower frequency arrivals, the AE signals are not apparent. In Fig. 4(a), the AE signals from the receiver $(\mathrm{M})$ at crack depths of $0.2,0.4,0.6$, and $0.8 \mathrm{~mm}$ are compared over a full $10 \mu$ s time scale. It can be seen that the arrival time of waves is about $0.7 \mu \mathrm{s}$, which is in good agreement with the theoretical arrival time of $0.69 \mu$ s calculated using the longitudinal wave velocity. The amplitude of the AE signal increases with the expansion of the crack depth. In Fig. 4(b), the AE signals filtered through a $20 \mathrm{kHz}$ high-pass filter are compared and found to be in good agreement with the experimental signal obtained by Prosser et al. ${ }^{(33)}$

Finally, AE signals are processed by FFT to obtain frequency spectra, as shown in Fig. 5(a). The variety law of signal frequency spectra under different crack lengths is compared. It can be observed that the frequency characteristics of AE signals are almost unchanged from the results of FFT analysis, and the energy is concentrated between $0-1000 \mathrm{kHz}$. In Fig. 5(b), the correlation of released energy and crack depth under different peak frequencies is plotted. The results of modal analysis show that the energy released by the growing crack is linearly proportional to crack depth. 


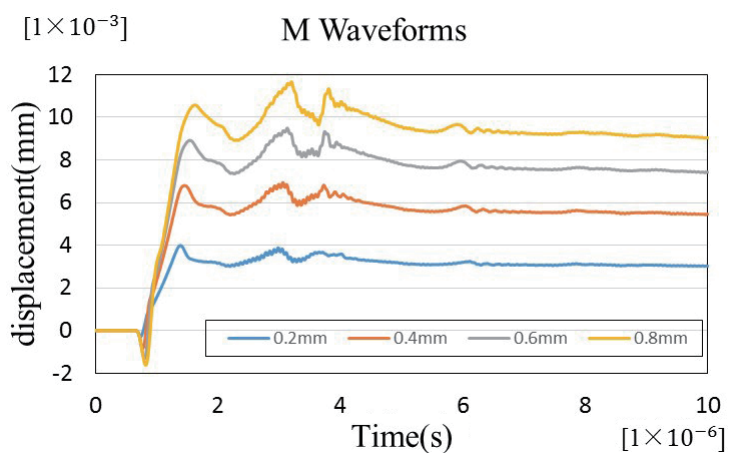

(a) $\left[1 \times 10^{-3}\right] \quad$ M Waveforms, $20 \mathrm{kHz}$ high pass

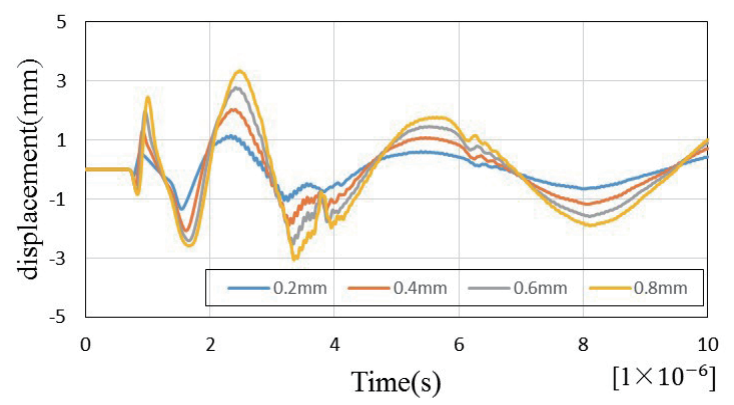

(b)

Fig. 4. (Color online) AE signals released by crack propagation in 316LN stainless steel at different crack depths. (a) AE signals detected by the receiver (M) at crack depths of $0.2,0.4,0.6$, and $0.8 \mathrm{~mm}$. (b) AE signals filtered through a $20 \mathrm{kHz}$ high-pass filter.

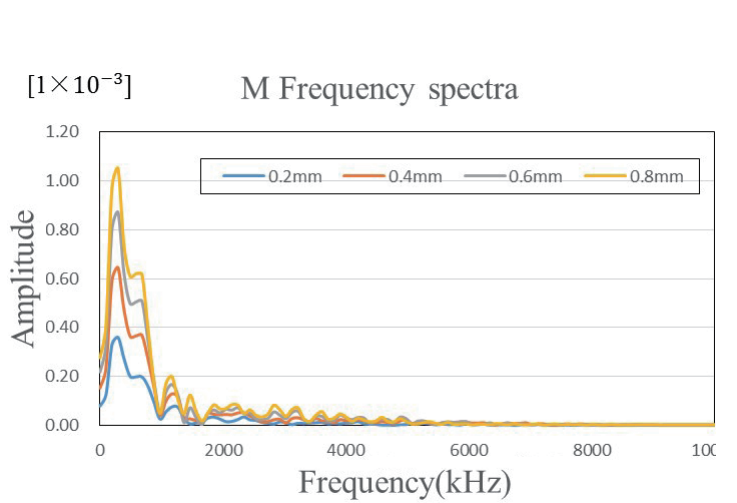

(a)

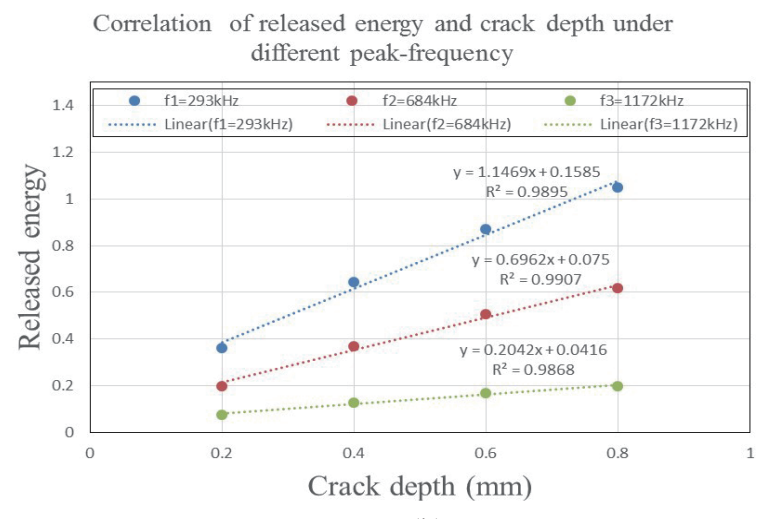

(b)

Fig. 5. (Color online) Spectral analysis of AE signals. (a) AE frequency spectra obtained by FFT of AE signals detected by the receiver $(\mathrm{M})$ at crack depths of $0.2,0.4,0.6$, and $0.8 \mathrm{~mm}$. (b) Correlation between energy released and crack depth under different peak frequencies.

\section{Conclusions}

In this study, AE signals generated by crack propagation at different crack depths in 316LN stainless steel were simulated. The energy released from the growing crack was linearly proportional to crack depth. The obtained trend regulation of the energy release rate of the AE source can be used to identify the AE source characteristic. Therefore, SCC initiation and propagation in $316 \mathrm{LN}$ stainless steel can be evaluated by this detection method. Furthermore, the appropriate $\mathrm{AE}$ sensors and detection systems have the potential to enable remote real-time monitoring of the initiation and propagation of SCC. In this study, the groove cracks based on the established model were analyzed. Complex cracks such as branch cracks will be evaluated in future work. 


\section{Acknowledgments}

This work is partially supported by the Fundamental Research Funds for the Central Universities (102472015148 \& 10247201551).

\section{References}

1 S. L. Chen, Y. T. Cheng, Z. W. Lin, and Y. Y. Chen: Sens. Mater. 27 (2015) 763.

2 P. J. S. Cruz, A. D. de Leon, and C. K. Y. Leung: Sens. Mater. 21 (2009) 65.

3 L. Zhang, X. Feng, M. Li, X. Wang, and F. Lin: Forging Stamping Tech. 39 (2014) 1.

4 C. Jomdecha, A. Prateepasen, and P. Kaewtrakulpong: NDT and E Int. 40 (2007) 584.

5 A. Davis, R. C. Bush, J. C. Harvey, and M. F. Foley: SID Symp. Digest Tech. Pap. 32 (2001) 934.

6 H. Chang, E. Han, J. Q. Wang, and W. Ke: J. Mater. Sci. 40 (2005) 5669.

7 H. Sidhom, T. Amadou, H. Sahlaoui, and C. Braham: Metall. Mater. Trans. A 38 (2007) 1269.

8 A. Arora: Corrosion 40 (1984) 459.

9 Y. P. Kim, M. Fregonese, H. Mazille, D. Féron, and G. Santarini: NDT and E Int. 36 (2003) 553.

10 J. Kovač, A. Legat, B. Zajec, T. Kosec, and E. Govekar: Ultrasonics 62 (2015) 312.

11 L. Calabrese, L. Bonaccorsi, M. Galeano, E. Proverbio, D. D. Pietro, and F. Cappuccini: Corros. Sci. 98 (2015) 573.

12 J. Kova, M. Leban, and A. Legat: Mater. Corros. 58 (2007) 970.

13 J. B. Coble, P. Ramuhalli, R. M. Meyer, and H. Hashemian: IEEE Instrum. Meas. Mag. 16 (2013) 32.

14 S. Ramadan, L. Gaillet, C. Tessier, and H. Idrissi: Appl. Surf. Sci. 254 (2008) 2255.

15 K. Aki and P. G. Richards: Quantitative Seismology (Univ. Science Books, Sausalito, 2002).

16 S. Caines, F. Khan, Y. Zhang, and J. Shirokoff: J. Loss Prev. Process Ind. 47 (2017) 72.

17 A. M. Homborg, R. A. Cottis, and J. M. C. Mol: Electrochimica Acta 222 (2016) 627.

18 Y. Hou, C. Aldrich, K. Lepkova, L. L. Machuca, and B. Kinsella: Corros. Sci. 112 (2016) 63.

19 R. Vidya Sagar: Case Stud. Constr. Mater. 6 (2017) 162.

20 L. Yang, Z. C. Zhong, J. You, Q. M. Zhang, Y. C. Zhou, and W. Z. Tang: Surf. Coat. Technol. 232 (2013) 710.

21 W. Hwang, S. Bae, J. Kim, S. Kang, N. Kwag, and B. Lee: Nucl. Eng. Technol. 47 (2015) 454.

22 A. Zaki, H. K. Chai, A. Behnia, D. G. Aggelis, J. Y. Tan, and Z. Ibrahim: Constr. Build. Mater. 136 (2017) 609.

23 F. Q. Su, K. I. Itakura, G. Deguchi, and K. Ohga: Appl. Energy 189 (2017) 142.

24 H. Wang, D. Liu, Z. Cui, C. Cheng, and Z. Jian: Theor. Appl. Fract. Mech. 85 (2016) 283.

25 A. Danyuk, I. Rastegaev, E. Pomponi, M. Linderov, D. Merson, and A. Vinogradov: Procedia Eng. 176 (2017) 284.

26 T. Suzuki, T. Shiotani, and M. Ohtsu: Constr. Build. Mater. 136 (2017) 619.

27 Y. Shao, Y. Yu, Y. Zhang, S. Wei, and X. Li: Proc. 10th Int. Conf. Sensing Technology (ICST 2016) No. 1570284226 .

28 K. Grabowski, M. Gawronski, I. Baran, W. Spychalski, W. J. Staszewski, T. Uhl, T. Kundu, and P. Packo: Ultrasonics 68 (2016) 142.

29 S. Patil, B. Karkare, and S. Goyal: Constr. Build. Mater. 68 (2014) 326.

30 Y. Kawasaki, T. Wakuda, T. Kobarai, and M. Ohtsu: Constr. Build. Mater. 48 (2013) 1240.

31 H. A. Elfergani, R. Pullin, and K. M. Holford: Constr. Build. Mater. 40 (2013) 925.

32 M. M. Sherif, J. Tanks, and O. E. Ozbulut: Cem. Concr. Res. 95 (2017) 178.

33 W. H. Prosser, M. A. Hamstad, J. Gary, and A. O’Gallagher: J. Acoust. Emission 17 (1999) 37. 


\section{About the Authors}

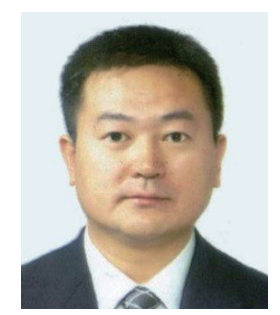

Xuefeng Li graduated from Shenyang Institute of Engineering, China, in 1999. He received his M.E. and D.E. degrees from Fukuoka Institute of Technology, Fukuoka, Japan, in 2004 and 2007, respectively. From 2007 to 2013, he was a postdoctorate and assistant researcher at Waseda University, Kitakyushu, Japan. Since 2010, he has been an associate professor at Tongji University. His research interests are in sensors and NDT.

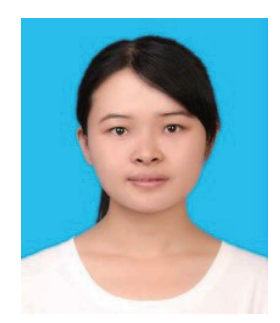

Yujiao Shao received her B.S. degree in measuring and control instruments from China JiLiang University, Hangzhou, China, in 2014. She is currently working toward an M.Sc. degree in control theory and control engineering at Tongji University, Shanghai, China. Her current research interests include acoustic emission and material defect detection.

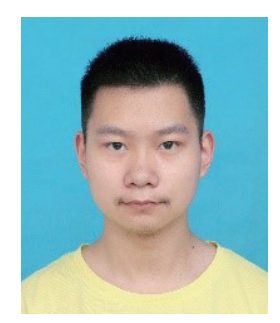

Yuan Yu received his B.S. degree from Jiangnan University, China, in 2014. Since 2014, he has been studying at Tongji University for his M.S. degree. His research interests are in sensors and NDT.

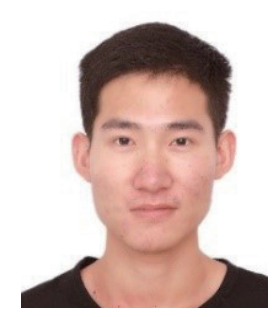

Yin Zhang received his B.S. degree from Tongji University, China. Since 2015, he has been studying at Tongji University for his M.S. degree. His research interests are in sensors and NDT.

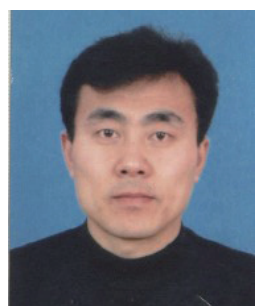

Runjie Shen received his B.S. degree from Xi'an Polytechnic University, China, in 1995. His M.S. degree was received Xi'an Jiaotong University, China, in 1998 and his Ph.D. degree from Zhejiang University, China, in 2004. From 2004 to 2006, he was a postdoctorate at Zhejiang University. In 2006, he became an associate researcher at Zhejiang University. Since 2011, he has been an associate researcher at Tongji University. His research interests are in sensors and NDT. 